Mathematical Research Letters 8, 1-13 (2001)

\title{
CONSTRUCTING HYPERBOLIC MANIFOLDS WHICH BOUND GEOMETRICALLY
}

\author{
D. D. LONG ${ }^{1}$ AND A. W. REID ${ }^{2}$
}

\section{Introduction}

Let $\mathbf{H}^{n}$ denote hyperbolic $n$-space, that is the unique connected simply connected Riemannian manifold of constant curvature -1 . By a hyperbolic $n$ orbifold we shall mean a quotient $\mathbf{H}^{n} / \Gamma$ where $\Gamma$ is a discrete group of isometries of $\mathbf{H}^{n}$. If a hyperbolic $n$-manifold $M$ is the totally geodesic boundary of a hyperbolic $(n+1)$-manifold $W$, we will say that $M$ bounds geometrically. It was shown in [11] that if a closed orientable hyperbolic $M^{4 k-1}$ bounds geometrically, then $\eta\left(M^{4 k-1}\right) \in \mathbf{Z}$. Closed hyperbolic 3-manifolds with integral eta are fairly rare - for example, of the 11,000 or so manifolds in the census of small volume closed hyperbolic 3-manifolds, computations involving Snap (see [3]) rule out all but 41. (We refer the reader to [24] which contains the list of manifolds in the census with Chern-Simons invariant zero, as well as which of these have integral eta.)

Hyperbolic 3-manifolds with totally geodesic boundary are fairly easily constructed given the Hyperbolization Theorem of Thurston [20], but to the authors' knowledge, there was only one known prior example of a closed hyperbolic $n$ manifold (with $n \geq 3$ ) which bounded geometrically, a somewhat ad hoc construction which appears in [18], based on a hyperbolic 4-manifold example due to Davis [4]. The difficulty is that almost nothing is known about hyperbolic manifolds in dimensions $\geq 4$; some constructions exist (see [5], [6], [7]) but they do not appear to be sufficient to address this problem.

This paper ameliorates this situation somewhat by providing a construction of examples in all dimensions. We show,

Theorem 1.1. Let $M$ be a nonorientable closed hyperbolic n-orbifold which can be immersed totally geodesically into a closed orientable hyperbolic $(n+1)$-orbifold $W$.

Then $M$ has a finite covering which is a manifold that bounds geometrically.

As we show using arithmetic techniques, infinitely many commensurability classes of such examples in all dimensions may readily be constructed. It is worth pointing out that although using the separability of $\pi_{1}(M)$ in $\pi_{1}(W)$ (see

Received April 24, 2001.

1 This work was partially supported by the N. S. F. .

2 This work was partially supported by the N. S. F. and The Alfred P. Sloan Foundation. 
[10]) easily promotes some finite covering of $M$ to an embedded submanifold of a hyperbolic manifold, nothing in the proof of that theorem gives the control necessary to guarantee that this embedding will be separating. The proof of Theorem 1.1 uses more subtle considerations. In particular, we prove the following which is perhaps of independent interest.

Theorem 1.2. Let $X$ be a nonorientable finite volume hyperbolic n-orbifold.

Then $X$ has a nonorientable finite sheeted manifold covering.

This paper is organized as follows. We begin by proving a simple lemma, 2.2, which finds a carefully controlled torsion-free subgroup of finite index; we then show using subtle algebraic considerations that one can construct representations of the required type (Theorem 2.6 and Theorem 2.8). The algebraic and geometric ingredients are assembled to prove the main theorem in Theorem 3.1.

The authors thank the referee for his careful and thorough reading of the first version of this manuscript.

\section{Preliminaries}

We collect a few basic facts about finite co-volume groups of hyperbolic isometries as well as some group theoretic lemmas which we shall use.

2.1. Recall that the full group of isometries of $\mathbf{H}^{n}$ can be identified with $\mathrm{O}_{0}\left(f_{n} ; \mathbf{R}\right)$ where $f_{n}$ is the quadratic form $<1,1, \ldots 1,-1>$. The group of orientation-preserving isometries of $\mathbf{H}^{n}$ is a subgroup of index 2 in $\mathrm{O}_{0}\left(f_{n} ; \mathbf{R}\right)$ consisting of those elements of determinant 1 , denoted by $\mathrm{SO}_{0}\left(f_{n} ; \mathbf{R}\right)$.

We have the following consequence of Mostow Rigidity ([16]):

Theorem 2.1. Let $W=\mathbf{H}^{n} / \Gamma$ be a finite volume hyperbolic $n$-orbifold with $n \geq 3$. Then we can conjugate $\Gamma$ in $\mathrm{O}_{0}\left(f_{n} ; \mathbf{R}\right)$ so that $\Gamma$ has entries in a number field.

A number field $L$ as in the conclusion of Theorem 2.1 is called a field of definition for $\Gamma$. As is shown in [21] there is a minimal such field of definition.

2.2. The next lemma is the main tool that we use to carefully choose a torsionfree subgroup of finite index.

Lemma 2.2. Let $G$ be a group admitting epimorphisms $\theta_{i}: G \rightarrow F_{i}, i=1,2$ each of which has torsion-free kernel. Define a homomorphism

$$
\Theta=\theta_{1} \times \theta_{2}: G \rightarrow F_{1} \times F_{2}
$$

and consider an element $\left(\alpha_{1}, \alpha_{2}\right) \in F_{1} \times F_{2}$ with $\alpha_{1}$ having order $2^{k_{1}} \mathrm{~m}, \alpha_{2}$ having order $2^{k_{2}}$ where $m$ is odd and $k_{1}<k_{2}$. Denote the finite cyclic group generated by this element by $<\left(\alpha_{1}, \alpha_{2}\right)>$.

Then $\Theta^{-1}<\left(\alpha_{1}, \alpha_{2}\right)>$ is torsion-free. 
Proof. The key claim here is that if one chooses any element $\xi$ of the subgroup $<\left(\alpha_{1}, \alpha_{2}\right)>$, then there is always a power $\xi^{s}$ for which one factor is the identity and the other factor is not. Using the fact that the homomorphisms $\theta_{i}$ had torsion free kernel, this will imply that $\xi$ cannot have been the image of a finite order element.

The claim is proved as follows. Suppose that $\xi=\gamma^{r}$ is the $r$-th power of the generator $\gamma=\left(\alpha_{1}, \alpha_{2}\right)$ where we may assume that $r \mid 2^{k_{2}} m$. If $r=2^{a} n$ where $n$ divides $m$ then we reduce to the case when $r=2^{a} m$ by raising $\gamma^{r}$ to the power $m / n$.

Now suppose that $k_{1} \leq a<k_{2}$. Then $\gamma^{2^{a} m}$ gives rise to an element which is the identity in the first component and not the second, as required. If $a<k_{1}<$ $k_{2}$, then the element has order $2^{k_{1}-a}$ in the first component and $2^{k_{2}-a}$ in the second and since $k_{1}-a<k_{2}-a$, taking a further power proves the result in this case also.

The proof of the lemma actually shows a more general statement, namely, if $\alpha_{1}$ has order $k_{1}$ and $\alpha_{2}$ has order $k_{2}$ and any prime which divides $G C D\left(k_{1}, k_{2}\right)$ occurs with different multiplicity in $k_{1}$ and $k_{2}$, then the same conclusion holds. We only use the simplified version stated here. Much of the algebraic work of this paper is devoted to proving that representations to which Lemma 2.2 applies can be constructed; our main result in this direction can be (informally) stated:

Theorem 2.3. Let $\Gamma$ be a nonorientable finite co-volume hyperbolic n-orbifold group.

Then given an integer $K$, there is a homomorphism onto a finite group $p$ : $\Gamma \rightarrow A$ for which there is an orientation reversing element $\delta_{p} \in \Gamma$ of infinite order whose image has order $2^{k}$ for some $k>K$.

A more precise version (which involves more terminology) is given in Theorem 2.8. We also need the following which goes back to Minkowski, for which we fix some notation.

Notation: Throughout, if $k$ is a number field then $R_{k}$ will denote the ring of integers in $k, R_{S}=R_{k}[S]$ will denote a subring of $k$ where a finite number of $k$-primes $S$ are inverted. Also if $\nu$ is a place of $k$ then completion will be denoted by a subscript $\nu$. The ring of $\nu$-adic integers of $k_{\nu}$ will be denoted $R_{\nu}$.

Lemma 2.4. Let $L$ be a number field, $S$ be a finite collection of prime ideals in $R_{L}$, and $\wp \subset R_{S}$ be a prime ideal lying over the rational prime $p \neq 2$.

Then $\operatorname{Ker}\left\{\pi_{\wp}: \mathrm{GL}\left(n, R_{S}\right) \rightarrow \mathrm{GL}\left(n, R_{S} / \wp\right)\right\}$ contains no q-torsion for any primes $q$ not divisible by $\wp$.

Proof. We begin with some general comments. It will be convenient to pass to the completions of $L$ and $R_{S}$, since these are principal ideal domains where unique factorization is particularly simple (see [9] for details). For a place $\nu$ we have injections $L \hookrightarrow L_{\nu}$ and $R_{S} \hookrightarrow R_{S, \nu}$ which induce a homomorphism $\mathrm{GL}\left(n, R_{S}\right) \hookrightarrow \mathrm{GL}\left(n, R_{S, \nu}\right)$. Note also that $R_{S, \nu}=R_{\nu}$ away from the places $\nu$ 
associated to primes in $S$. Let $x_{\nu}$ be a uniformizer for $L_{\nu}$ so that every element in $L_{\nu}$ has a unique expression as $x_{\nu}^{m} u$ for an integer $m$ and $\nu$-adic unit $u$.

Using the ring homomorphism $R_{\nu} \rightarrow R_{\nu} /<x_{\nu}>$ there are obvious reduction homomorphisms induced from $\mathrm{GL}\left(n, R_{\nu}\right) \rightarrow \mathrm{GL}\left(n, R_{\nu} /<x_{\nu}>\right)$, and composition of inclusion and this reduction map is an equivalent definition of the homomorphisms $\pi_{\wp}$, where $\nu$ is the place associated to the prime ideal $\wp$.

Now fix $x$ to be a uniformizer for our specific prime $\wp$, and suppose that $\tau$ is an element of $\operatorname{GL}\left(n, R_{S}\right)$ prime order $q$.

The element $\tau \in \operatorname{Ker}\left(\pi_{\wp}\right)$ can be written $\tau=I+x^{t} T$ for $t \in \mathbf{Z}^{+}$and a matrix $T \in M\left(n, R_{\nu}\right)$ whose entries are not all divisible by $x$.

By the binomial theorem

$$
\tau^{q}=I=I+q x^{t} T+\frac{q(q-1)}{2} x^{2 t} T^{2}+\mathrm{O}\left(x^{3 t}\right)
$$

so that

$$
q T+\frac{q(q-1)}{2} x^{t} T^{2}=0 \bmod x^{2 t} M\left(n, R_{\nu}\right)
$$

and hence

$$
q T=0 \bmod x^{t} M\left(n, R_{\nu}\right) .
$$

If $x$ does not divide $q$ then $T=0 \bmod x$ which is false.

Remark. Notice that there is a bound to the order of an element of finite order in $\operatorname{GL}(n, L)$ for $L$ a number field. This follows from the fact that the number of roots of unity of bounded degree over $\mathbf{Q}$ is finite.

2.3. We include here a result that follows from work of Weisfeiler [23] or Nori [12]. We give a proof in $\S 5$ as it seems to us worthwhile to record this explicitly for groups of hyperbolic isometries.

We begin with a discussion of some notation and results from the theory of simple groups arising from orthogonal groups. Let $f$ be an $m$-dimensional quadratic form over the finite field $\mathbf{F}$ of cardinality $q=p^{n}$. To simplify some of the discussion we assume $p$ is odd. In the case when $m$ is also odd, there is a unique orthogonal group $\mathrm{O}(m, q)$ up to isomorphism, and when $m$ is even there are two $\mathrm{O}_{ \pm}(m, q)$ (see [19] p 377 Theorem 5.8). Let $\mathrm{SO}(m, q)$ and $\mathrm{SO}_{ \pm}(m, q)$ denote the special orthogonal groups in these cases. We remark that since the order of these orthogonal and special orthogonal groups are divisible by large powers of $p$ (see [19] pp 375-382), these groups do contain elements of order $p$.

Let $\Omega(m, q)=[\mathrm{O}(m, q), \mathrm{O}(m, q)]$ when $m$ is odd (resp. $\Omega_{ \pm}(m, q)=$ $\left[\mathrm{O}_{ \pm}(m, q), \mathrm{O}_{ \pm}(m, q)\right]$ when $m$ is even) where $[G, G]$ denotes the commutator subgroup of a group $G$. When $m$ is even $\Omega_{ \pm}(m, q)$ has index 2 in $\mathrm{SO}_{ \pm}(m, q)$ and has a center of order 1 or 2 . Let $\mathrm{P} \Omega_{ \pm}(m, q)$ be the central quotient group.

We summarize the important facts for us in the following theorem (see [19] pp. $383-384$ for a discussion):

\section{Theorem 2.5.}

1. When $m$ is odd, $\Omega(m, q)$ is a simple subgroup of $\mathrm{O}(m, q)$ of index 4. 
2. When $m$ is even, $\Omega(m, q)$ is a subgroup of $\mathrm{O}(m, q)$ of index 4 and the quotient $\mathrm{P} \Omega_{ \pm}(m, q)$ is simple whenever $m \geq 6$.

3. When $m=4, \mathrm{P} \Omega_{+}(4, q) \cong \operatorname{PSL}(2, q) \times \operatorname{PSL}(2, q)$ and $\operatorname{P} \Omega_{-}(4, q) \cong \operatorname{PSL}\left(2, q^{2}\right)$.

Notation: We sometimes suppress the subscripts \pm , and also use the notation $\Omega(f ; q)$, or $\Omega(f ; \mathbf{F})$ where $\mathbf{F}$ is a finite field, or simply just $\Omega$ when no confusion will arise.

Now assume $\Gamma$ is a finite co-volume subgroup of $\mathrm{O}_{0}\left(f_{n} ; \mathbf{R}\right)$. By Theorem 2.1 we can assume that $\Gamma$ is a subgroup of $\mathrm{O}_{0}\left(f_{n} ; L\right)$ for $L$ the minimal field of definition. Now $\Gamma$ is finitely generated so that $\Gamma<\mathrm{O}_{0}\left(f_{n} ; R\right)$ for a subring $R \subset L$ where a finite number of primes of $R_{L}$ are inverted. As in Lemma 2.4, apart from a finite number of primes we have homomorphisms $\pi_{\wp}: \mathrm{O}\left(f_{n} ; R\right) \rightarrow \mathrm{O}\left(f_{n} ; R / \wp\right)$. These target groups are simply certain of the finite orthogonal groups which we have discussed above. We require a generalization of The Strong Approximation Theorem [15] for arithmetic groups to handle subgroups $\Gamma$. The generalization we want is ([12] and [23]):

Theorem 2.6. In the notation above, let $q$ denote the cardinality of the residue class field $R_{L} / \wp$. Then for all but a finite number of primes $\wp$, we have

1. $\Omega(n+1, q) \leq \pi_{\wp}(\Gamma) \leq \mathrm{O}(n+1 ; q)$, when $n+1$ is odd.

2. $\mathrm{P} \Omega_{ \pm}(n+1, q) \leq \mathrm{P} \pi_{\wp}(\Gamma) \leq \mathrm{PO}_{ \pm}(n+1 ; q)$, when $n+1$ is even (where the above notation indicates that only the correct sign is chosen in the subscript).

We shall prove Theorem 2.6 in $\S 5$.

2.4. We include an alternative description of the groups $\Omega(m, q)$ that will be useful for us (see [14] Chapters V and VI).

Assume that $\mathbf{F}$ is a finite field of odd characteristic. In this case $\mathbf{F} / \mathbf{F}^{* 2}$ has order 2. Let $(V, f)$ be a non-zero regular quadratic space over $\mathbf{F}$. Any reflection in $\mathrm{O}(f ; \mathbf{F})$ is determined by a vector $v \in V$, and acts by reflecting in the hyperplane orthogonal to $v$ (with respect to the bilinear form $B$ defined by $f)$. Denote such a reflection by $\tau_{v}$. Any element $\tau$ of $\mathrm{O}(f ; \mathbf{F})$ is a product of such reflections, say $\tau=\tau_{v_{1}} \ldots \tau_{v_{r}}$ and using this we can define a homomorphism, the spinor norm:

$$
\theta: \mathrm{O}(f ; \mathbf{F}) \rightarrow \mathbf{F} / \mathbf{F}^{* 2}
$$

by

$$
\theta\left(\tau_{v_{1}} \ldots \tau_{v_{r}}\right)=f\left(v_{1}\right) \ldots f\left(v_{r}\right) \bmod \mathbf{F}^{* 2} \in \mathbf{F} / \mathbf{F}^{* 2} .
$$

That this is independent of the choice of how $\tau$ is written as a product of reflections is discussed in $[14] \S 55$.

Restricting $\theta$ to $\mathrm{SO}(f ; \mathbf{F})$ it can be shown that $\left.\operatorname{Ker} \theta\right|_{\mathrm{SO}_{(f ; \mathbf{F})}}=\Omega([14])$. Thus a non-trivial coset representative for $\mathrm{SO}(f ; \mathbf{F}) / \Omega$ is any element whose spinor norm is a non-square in $\mathbf{F}$. We will need some additional control over such a representative. 
Any quadratic form $f$ (as above) in at least two variables represents all elements of $\mathbf{F}$, and hence given $e \in \mathbf{F}$ a non-square, we can find $v_{1} \in V$ with $f\left(v_{1}\right)=e$. Let $v_{2} \in V$ have $f\left(v_{2}\right)=1$. Let $\tau_{i}$ be the reflections determined by $v_{i}$ for $i=1,2$. Then $\tau=\tau_{1} \tau_{2} \in \mathrm{SO}(f ; \mathbf{F})$ is of spinor norm $e$, and hence does not lie in $\Omega$. One sees easily from the fact that the spinor kernel is the commutator subgroup that the quotient $\mathrm{O} / \Omega$ is a Klein 4-group so we may take any other reflection to generate the other element of order two in the quotient. Summarizing this discussion (see also [14]), we have

Corollary 2.7. Let $(V, f)$ be as above.

Then $\mathrm{O}(f ; \mathbf{F}) / \Omega \cong \mathbf{Z} / 2 \mathbf{Z} \oplus \mathbf{Z} / 2 \mathbf{Z}$ with coset representatives being $\left\{I, \tau_{1}, \tau_{1} \tau_{2}, \tau_{2}\right\}$.

2.5. As an application of Theorem 2.6 we now prove (where we assume as in $\S 2.1$ that $\Gamma$ consists of matrices with entries in the minimal field of definition $L$ ),

Theorem 2.8. Let $\Gamma$ be a nonorientable finite co-volume hyperbolic n-orbifold group. Then given an integer $K$, for infinitely many homomorphisms $\pi_{\wp}: \Gamma \rightarrow$ $\mathrm{O}\left(f_{n} ; q\right)$ there is an orientation reversing element $\delta_{\wp} \in \Gamma$ of infinite order whose image has order $2^{k}$ for some $k>K$.

This theorem requires the following lemma.

Lemma 2.9. Let $L$ be a number field, and $R=R_{S}$ for some finite collection of L-primes $S$. Given an integer $K$, then for infinitely many primes $\wp \subset R$ the groups $\mathrm{O}\left(f_{n} ; R / \wp\right)$ contain an element with determinant -1 and of order $2^{k}$ for some $k>K$.

Proof. It suffices to exhibit such an element in $\mathrm{O}(3 ; \mathbf{F})$ for infinitely many fields $\mathbf{F}=R / \wp$. To see this, note first that from Theorem 2.5 in the case of finite fields of odd characteristic, the groups $\mathrm{O}\left(f_{3} ; \mathbf{F}\right)$ and $\mathrm{O}(3 ; \mathbf{F})$ are isomorphic. Furthermore by considering the form $f_{n}=<1 \ldots, 1>\oplus f_{3}$, we see that there is a copy of $G=\mathrm{O}\left(f_{3} ; R / \wp\right)<\mathrm{O}\left(f_{n} ; R / \wp\right)$. We shall exhibit an element $g \in G$ of order $2^{k}$, then one of the elements $< \pm 1,1, \ldots, 1>\oplus g$ is the required element.

To construct $g$ consider the cyclic groups $C_{k}=<y \mid y^{2^{k}}=1>$. Such groups are subgroups of $\mathrm{O}(3)=\mathrm{O}(3 ; \mathbf{R})$ and by conjugating in $\mathrm{O}(3)$ we can assume that $C_{k}$ consists of matrices whose entries lie in the ring of integers $R_{k}$ of the number field $N_{k}$. Now Lemma 2.4 applies in this case to inject the cyclic groups into all but a finite number of the groups $\mathrm{O}\left(3 ; R_{k} / P\right)$ where $P$ is a prime ideal of $R_{k}$. Hence we have the element of order $2^{k}$ in these orthogonal groups.

To get an element in those orthogonal groups arising from quotients obtained by reducing by primes of $L$, we simply choose infinitely many primes that split completely in both $L$ and $N_{k}$. The existence of such a family follows by taking the number field $U$ generated over $\mathbf{Q}$ by $L$ and $N_{k}$. By standard properties of ramification of primes in field extensions, if a prime $p$ splits completely in $U$ it will split completely in the subfields $L$ and $N_{k}$. It is a well-known consequence of how prime ideals behave in finite extensions of $\mathbf{Q}$, that there are infinitely 
many rational primes that split completely in the finite extension $U / \mathbf{Q}$, see [13] Theorem 4.12 for example. It then follows that for these infinitely many primes the residue class fields obtained are isomorphic. This completes the proof.

Proof of Theorem 2.8. In the notation established above, by Lemma 2.9, for infinitely many primes $\wp$ with residue field of odd cardinality $q$, the groups $\mathrm{O}\left(f_{n} ; q\right)$ contain elements of large 2-power order and of determinant -1 , so that if $\pi_{\wp}$ is a surjection, then we are done.

Otherwise, we argue as follows. Let

$$
\chi: \mathrm{O}\left(f_{n} ; q\right) \rightarrow \mathrm{O}\left(f_{n} ; q\right) / \Omega\left(f_{n}, q\right)
$$

denote the projection homomorphism. From Corollary 2.7, this quotient group is the Klein 4-group.

Since we are assuming that $\pi_{\wp}$ does not surject, and since by hypothesis $\Gamma$ contains an element of determinant -1 , it follows from Theorem 2.6 that the index of $\pi_{\wp}(\Gamma)$ in $\mathrm{O}\left(f_{n} ; q\right)$ is precisely two. Our strategy will be to exhibit in this image an element of order two which comes from an orientation reversing element and has centralizer containing an element of large 2-power order.

The existence of the element of determinant -1 in $\pi_{\wp}(\Gamma)$ together with Corollary 2.7 implies that $\chi \pi_{\wp}(\Gamma)$ must contain either the image of $\tau_{1}$ or $\tau_{2}$ (in the notation of $\S 2.3)$. Since $\pi_{\wp}(\Gamma)$ contains every element of $\Omega\left(f_{n}, q\right)$ up to sign (the signs only being necessary in the even dimensional case), it follows that at least one of the elements $\pm \tau_{1}, \pm \tau_{2}$ must actually belong to $\pi_{\wp}(\Gamma)$. In any of these cases, the centralizer of this element contains a group isomorphic to $\mathrm{O}(3, q)$ and since these latter groups contain elements of large 2-power order (cf. the proof Lemma 2.9) this completes the proof.

\section{Proof of Theorem 1.1}

The goal of this section is to prove the main theorem of the introduction:

Theorem 3.1. Let $M$ be a nonorientable closed hyperbolic n-orbifold which can be immersed totally geodesically into a closed orientable hyperbolic $(n+1)$-orbifold $W$.

Then $M$ has a finite (manifold) covering which bounds geometrically.

We show in $\S 4$, that using arithmetic considerations one can construct infinitely many such examples.

To prove the theorem we shall promote the immersed non-orientable hyperbolic $n$-manifold to an embedded non-orientable hyperbolic $n$-manifold in a hyperbolic $(n+1)$-manifold which is a finite cover of $W$. Achieving this proves the theorem for we have,

Lemma 3.2. Suppose that $M$ is a codimension 1 nonorientable embedded totally geodesic submanifold of a closed orientable hyperbolic manifold.

Then the orientation cover of $M$ bounds geometrically. 
Proof. Such a submanifold determines a canonical map $\pi_{1}(W) \rightarrow \mathbf{Z} / 2 \mathbf{Z}$, for example by taking the corresponding element of $H^{1}(W ; \mathbf{Z} / 2 \mathbf{Z})$ and the corresponding covering $p: W_{2} \rightarrow W$ contains the orientation cover $M_{2}$ of $M$ as a separating totally geodesic submanifold. Splitting $W_{2}$ along $M_{2}$ exhibits $M_{2}$ as a geometric boundary.

Remark. It is built into the construction $\eta\left(M_{2}\right)=0$ and so the obstruction of [11] (in dimensions $4 k-1$ ) vanishes.

Proof of Theorem 3.1. To find the finite cover of $W$ referred to above, we begin by exhibiting an infinite order element of $\pi_{1}(M)$ which is orientation reversing and lies in a torsion-free subgroup of finite index in $\pi_{1}(W)$.

Let $W=\mathbf{H}^{n+1} / \Gamma$ with $\Gamma<\operatorname{SO}_{0}\left(f_{n+1} ; L\right)$ and where by Theorem 2.1 we can arrange that $L$ is a number field. Since $M$ is immersed in $Q$ as a totally geodesic suborbifold, we can find a totally geodesic copy of $\mathbf{H}^{n}$ in $\mathbf{H}^{n+1}$, which we denote by $\mathbf{H}$ and a group of isometries $\Delta$ of $\mathbf{H}$ with $\mathbf{H} / \Delta \hookrightarrow \mathbf{H}^{n+1} / \Gamma$. We can assume that $\Delta$ is the maximal subgroup of $\Gamma$ preserving $\mathbf{H}$, and by a further conjugacy (of $\Gamma$ ) if necessary we can embed $\Delta$ in $\Gamma$ as a group of isometries of the form $<1>\oplus f_{n}$, where the coefficents of $\Delta$ now lie in the minimal field of definition of $\Delta$. Note that since $\Gamma<\mathrm{SO}_{0}\left(f_{n+1} ; L\right)$ a conjugacy of $\Delta$ to preserve the form $<1>\oplus f_{n}$ is defined over a number field, so that we now have $\Gamma<\mathrm{SO}_{0}\left(f_{n+1} ; L_{1}\right)$, where $L_{1}$ is a finite extension of $L$. Indeed $\Gamma<\mathrm{SO}_{0}\left(f_{n+1} ; R\right)$ for some subring $R \subset L_{1}$ where a finite collection $S$ of primes of $L_{1}$ are inverted. Now $L_{1}$ need not be the minimal field of definition of $\Gamma$, but it is the location of $\Delta$ that is important for us. To avoid clutter of notation we abuse notation and just use $R$ for the ring of coefficients of $\Delta$.

The torsion in $\Gamma$ has bounded size (either from the above remark after Lemma 2.4 or from the fact that $\Gamma$ is cocompact [17]). Now from Theorem 2.8 and Lemma 2.4 we may assume that for an infinite number of primes $\wp$ the homomorphism $\pi_{\wp}$ restricted to $\Delta$ (with image in $\mathrm{O}\left(f_{n} ; R / \wp\right)$ ) satisfies the conclusion of Theorem 2.8.

We claim that we may now arrange a homomorphism of the type required by Lemma 2.2. To this end, choose any reduction map $\pi_{\wp}$ (as above) restricted to ( $\Gamma$ and) $\Delta$ and consider the order of the quotient group $\mathrm{O}\left(f_{n} ; R / \wp\right)$. Let $2^{a}$ be the maximal power of 2 which divides the order $\left|\mathrm{O}\left(f_{n} ; R / \wp\right)\right|$.

By Theorem 2.8, we may find another prime $\wp^{*}$ so that $\Delta$ contains an orientation-reversing element $\delta_{\wp^{*}}$ of infinite order whose image in the group $\mathrm{O}\left(f_{n} ; R / \wp^{*}\right)$ is an element of determinant -1 and of order $2^{k_{2}}$ where $k_{2}>a$. Moreover, when we consider the projection of $\delta_{\wp^{*}}$ into $\mathrm{O}\left(f_{n} ; R / \wp\right)$, it has order $2^{k_{1}} m$ where $k_{1} \leq a<k_{2}$. Thus the hypotheses of Lemma 2.2 are satisfied, and we may pass to a torsion-free subgroup $\Gamma_{0}$ of finite index in $\Gamma$ which contains the orientation reversing element $\delta_{\wp^{*}}$. It now follows that the preimage of $M$ in this covering will be a nonorientable totally geodesic hyperbolic $n$-manifold $M_{0}$ immersed in $W_{0}=\mathbf{H}^{n+1} / \Gamma_{0}$. Using the fact that $M_{0}$ is totally geodesic (the only place where this is used) there is a further finite sheeted covering of $W$ to which 
$M$ has a preimage which is an embedding, see [10]. Renaming, we are now in the situation of having an embedded nonorientable totally geodesic $n$-submanifold $M$ of a closed orientable hyperbolic $(n+1)$-manifold, $W$. Appealing to Lemma 3.2 completes the proof.

Remark. The proof is easily modified to give Theorem 1.2 of the introduction. Here is a sketch: Consider the discrete faithful representation $\rho: \pi_{1}(X) \rightarrow$ $\mathrm{O}\left(f_{n} ; R\right)$, where $R$ is a finitely generated integral domain coming from some ring of integers with a finite number of primes inverted. $X$ is nonorientable so there are some infinite order elements of determinant -1 . Choose prime reductions as above with torsion free kernel so that the hypotheses of Lemma 2.2 hold and the proof now proceeds exactly as in 3.1; the higher dimensional manifold plays a minor role at this stage of the proof.

\section{Examples}

There are two natural settings where the hypothesis of Theorem 1.1 hold, namely arithmetic subgroups of hyperbolic isometries and reflection groups. Recall the following construction of arithmetic subgroups of $\mathrm{O}_{0}\left(f_{n} ; \mathbf{R}\right)$.

Assume that $k$ is a totally real number field, let $f$ be a form in $n+1$ variables with coefficients in $k$, and be equivalent over $\mathbf{R}$ to the form $f_{n}$. Furthermore, if $\sigma: k \rightarrow \mathbf{R}$ is a field embedding, then the form $f^{\sigma}$ obtained by applying $\sigma$ to $f$ is defined over the real number field $\sigma(k)$. We insist that for embeddings $\sigma \neq i d$, $f^{\sigma}$ is equivalent over $\mathbf{R}$ to the form in $(n+1)$-dimensions, of signature $(n+1,0)$. Since $f$ is equivalent over $\mathbf{R}$ to $f_{n}, \mathrm{SO}(f ; \mathbf{R})$ is conjugate in $\mathrm{GL}(n+1, \mathbf{R})$ to $\mathrm{SO}\left(f_{n} ; \mathbf{R}\right)$. Thus there is an invertible matrix $\Phi$ such that $\Phi \mathrm{SO}_{0}\left(f ; R_{k}\right) \Phi^{-1}<$ $\mathrm{SO}_{0}\left(f_{n} ; \mathbf{R}\right)$ defines an arithmetic subgroup (see [2] or [1]).

The group $\Phi \mathrm{SO}_{0}\left(f ; R_{k}\right) \Phi^{-1}$ is cocompact if and only if the form $f$ does not represent 0 non-trivially with values in $k$, see [2]. For our purposes it suffices to note that choosing a form defined over a totally real field of degree at least 2 with the properties above ensures that the group $\Phi \mathrm{SO}_{0}\left(f ; R_{k}\right) \Phi^{-1}$ is co-compact. The reason being that at the non-trivial Galois embeddings the form is definite and hence cannot represent 0 over $\mathbf{R}$. The Hasse-Minkowski Theorem (see [14] for instance) now implies the form cannot represent 0 globally over $k$.

Definition. We call an arithmetic subgroup of $\mathrm{O}_{0}\left(f_{n} ; \mathbf{R}\right)$ constructed in this way real arithmetic.

The class of real arithmetic groups is a proper subset of the class of (arithmetic groups/hyperbolic manifolds) in odd dimensions. For convenience we shall also suppress the conjugacy.

Theorem 4.1. Let $f$ be an $n+1$-dimensional quadratic form defined over a totally real field $k$ of degree at least 2 with the properties above. Suppose in addition that $\mathbf{H}^{n} / \mathrm{O}_{0}\left(f ; R_{k}\right)$ is a non-orientable hyperbolic n-orbifold.

Then there is a torsion-free real arithmetic subgroup $\Delta$ in $\mathrm{SO}_{0}\left(f ; R_{k}\right)$ for which $\mathbf{H}^{n} / \Delta$ bounds geometrically. 
We note that the hypothesis that $\mathbf{H}^{n} / \mathrm{O}_{0}\left(f ; R_{k}\right)$ is nonorientable is easily achieved, for example by taking a diagonal form; in this case the group $\mathrm{O}_{0}\left(f ; R_{k}\right)$ contains a reflection.

Proof of 4.1. By hypothesis $\mathbf{H}^{n} / \mathrm{O}_{0}\left(f ; R_{k}\right)$ is a non-orientable hyperbolic $n$ orbifold.

The form $q=<1>\oplus f$ is also defined over $k$, is of signature $(n+1,1)$ and has the right properties at the other embeddings of $k$. Thus $\mathrm{SO}_{0}\left(q ; R_{k}\right)$ is a real arithmetic (and cocompact by the remarks above) subgroup of $\mathrm{SO}_{0}\left(f_{n+1} ; \mathbf{R}\right)$. Furthermore $\mathbf{H}^{n+1} / \mathrm{SO}_{0}\left(q ; R_{k}\right)$ is orientable.

Notice that we can inject $\mathrm{O}_{0}\left(f ; R_{k}\right) \hookrightarrow \mathrm{SO}_{0}\left(q ; R_{k}\right)$ by mapping $g \in \mathrm{O}_{0}\left(f ; R_{k}\right)$ to

$$
\left(\begin{array}{c|c}
\rho(g) & 0 \\
0 & g
\end{array}\right)
$$

where $\rho: \mathrm{O}_{0}\left(f ; R_{k}\right) \rightarrow\{ \pm 1\}$ is the determinant map, so that the image has determinant 1 as is required for the image to be in $\mathrm{SO}(q)$. Now apply Theorem 1.1 to exhibit a group $\Delta$ as required.

Remark. Although this result proves for example, that there are infinitely many hyperbolic 3-manifolds which bound geometrically, the construction produces manifolds of very large volume. It would be interesting to know if any of the 41 census examples not obstructed by the eta-invariant do or do not bound in this sense.

The definition of real arithmetic groups and Theorem 4.1 easily imply:

Corollary 4.2. Let $\Gamma$ be a real arithmetic subgroup of $\mathrm{SO}_{0}\left(f_{n} ; \mathbf{R}\right)$. Then $\Gamma$ is commensurable with a torsion-free subgroup $\Delta$ for which $\mathbf{H}^{n} / \Delta$ bounds geometrically.

\section{Proof of Theorem 2.6}

Let $L$ be the minimal field of definition of $\Gamma$. Since any group of isometries of hyperbolic space contains a subgroup of index 2 consisting of elements preserving orientation, it suffices to assume that $\Gamma<\mathrm{SO}_{0}\left(f_{n} ; L\right)$ and establish the theorem in this case.

We begin with some preliminary remarks. The group $\mathrm{SO}\left(f_{n}\right)$ is a connected semisimple (complex) linear algebraic group defined over $\mathbf{Q}$, and hence over $L$. Indeed $\mathrm{SO}\left(f_{n}\right)$ is defined by polynomials with integral coefficients, and with this in mind, it will often be convenient to view $\mathrm{SO}\left(f_{n}\right)$ as a linear algebraic group without specifying a base field for the affine space; above it is $\mathbf{C}$, but we will have recourse to consider algebraic closures of $\mathbf{Q}_{p}$ and $\mathbf{F}_{p}$. We make no further comment on this or on reducing algebraic varieties and algebraic groups defined by integer polynomials modulo $p$ (see [15] Chaps 2 and 3 for more details). No confusion should arise. 
A useful tool in algebraic geometry is restriction of scalars (see [15]). In our setting, if we view $\mathrm{SO}\left(f_{n}\right)$ as an algebraic group defined over $L$, the restriction of scalars produces an algebraic group $G=\mathbf{R}_{L / \mathbf{Q}}\left(\mathrm{SO}\left(f_{n}\right)\right) \subset \mathrm{GL}(N, \mathbf{C})$ for some integer $N$ (in fact $N=n d$ where $d$ is the degree of $L$ over $\mathbf{Q}$ ) such that $G_{\mathbf{Q}} \cong \mathrm{SO}\left(f_{n} ; L\right)$ (see [15]). In particular, we induce a faithful rational representation of $\Gamma$ into $G_{R}$ where $R=\mathbf{Z}[S]$ and $S$ is a finite set of rational primes. Denote this group by $\Gamma^{\prime}$. Note that apart from these primes in $S$ (we also assume $2 \in S$ ) we have homomorphisms $\pi_{p}$ as in Lemma 2.4 with $\pi_{p}\left(\Gamma^{\prime}\right)<\operatorname{GL}\left(N, \mathbf{F}_{p}\right)$. The results of Nori [12] (or Weisfeiler [23]) give control over the image.

Let $A$ denote the $R$-points of the Zariski closure of $\Gamma^{\prime}$ in $\operatorname{GL}(N, \mathbf{Q})$. Note that since $\Gamma$ is of finite co-volume it is Zariski dense in $\operatorname{SO}\left(f_{n} ; L\right)$ (by Borel's Density Theorem, see [16] Chapter 5, for example), and so by definition of the restriction of scalars, the Zariski closure of $\Gamma^{\prime}$ in $\mathrm{GL}(N, \mathbf{Q})$ is $G_{\mathbf{Q}}$.

Let $A\left(\mathbf{F}_{p}\right)=\pi_{p}(A)$ (where this is defined). Then we have the following due to Nori ([12] Theorem 5.1). We use the notation that if $\mathbf{F}$ is a field of characteristic $p$, and $Q<\mathrm{GL}(s, \mathbf{F})$, then $Q^{+}$will denote the normal subgroup of $Q$ generated by its elements of order $p$.

Theorem 5.1. For all but finitely many primes $p$, we have

$$
A\left(\mathbf{F}_{p}\right)^{+} \subset \pi_{p}\left(\Gamma^{\prime}\right) \subset A\left(\mathbf{F}_{p}\right) .
$$

Now from the remarks above $A\left(\mathbf{F}_{p}\right)=G_{\mathbf{F}_{p}}$, thus we deduce

$$
G_{\mathbf{F}_{p}}^{+} \subset \pi_{p}\left(\Gamma^{\prime}\right) \subset G_{\mathbf{F}_{p}} .
$$

For a rational prime $p$ let $V^{(p)}$ denote the finite set of places of $L$ lying above $p$. The theory of completions (see [9]) gives $L \otimes_{\mathbf{Q}} \mathbf{Q}_{p} \cong \bigoplus_{\nu \in V^{(p)}} L_{\nu}$, and it follows that restriction of scalars induces (see [15]):

$$
G_{\mathbf{Z}_{p}} \cong \prod_{\nu \in V^{(p)}} \mathrm{SO}\left(f_{n} ; R_{\nu}\right)
$$

where $R_{\nu}$ is the ring of $\nu$-adic integers of $L_{\nu}$ with $x_{\nu}$ a uniformizer. The key point to observe now is

$$
G_{\mathbf{F}_{p}} \cong \prod_{\nu \in V^{(p)}} \mathrm{SO}\left(f_{n} ; R_{\nu} /<x_{\nu}>\right),
$$

which can be seen directly from how the algebraic group $G$ lies as a subgroup of $\mathrm{GL}(N)$-regardless of the base field of the affine space.

Denote this isomorphism by $\Phi_{p}$ and let

$$
\psi_{\nu}: \prod_{\nu \in V^{(p)}} \mathrm{SO}\left(f_{n} ; R_{\nu} /<x_{\nu}>\right) \longrightarrow \mathrm{SO}\left(f_{n} ; R_{\nu} /<x_{\nu}>\right)
$$

denote the projection homomorphism. Note that at all but a finite collection of places $\nu$ of $L$ completion induces a faithful representation of $\Gamma$ in $\operatorname{SO}\left(f_{n} ; R_{\nu}\right)$. Using this and embedding $\Gamma$ in the product of such groups, it follows that (by 
construction) $\pi_{\wp}(\Gamma)=\psi_{\nu}(\Gamma)$ where $\nu$ is the place associated to $\wp$. Similarly, for all but a finite number of primes $p \in \mathbf{Z}$ we can inject $\Gamma^{\prime}$ into $G_{\mathbf{Z}_{p}}$.

Since $\Phi_{p}$ is an isomorphism we deduce from Theorem 5.1 (and the subsequent remarks) that

$$
\Phi_{p}\left(G_{\mathbf{F}_{p}}^{+}\right)<\Phi_{p}\left(\pi_{p}\left(\Gamma^{\prime}\right)\right)<\Phi_{p}\left(G_{\mathbf{F}_{p}}\right),
$$

and so for all but a finite number of places $\nu$ we get:

$$
\mathrm{SO}\left(f ; R_{\nu} /<x_{\nu}>\right)^{+}<\psi_{\nu} \Phi_{p}\left(\pi_{p}\left(\Gamma^{\prime}\right)\right)<\mathrm{SO}\left(f ; R_{\nu} /<x_{\nu}>\right) .
$$

From the discussion above, $\pi_{\wp}(\Gamma)=\psi_{\nu} \Phi_{p}\left(\pi_{p}\left(\Gamma^{\prime}\right)\right)$ and so,

$$
\mathrm{SO}\left(f ; R_{\nu} /<x_{\nu}>\right)^{+}<\pi_{\wp}(\Gamma)<\mathrm{SO}\left(f ; R_{\nu} /<x_{\nu}>\right),
$$

When $f_{n}$ is not 4 -dimensional we are now done by Theorem 2.5 , where in the case of $n$ odd (so $n+1$ is even) we pass to the central quotient again.

For the case of $n=3$, so that the form is 4-dimensional, the above argument works in the case of $\operatorname{PSL}\left(2, q^{2}\right)$ which is simple. In the case of $\operatorname{PSL}(2, q) \times$ $\operatorname{PSL}(2, q)$ it is well known that $\operatorname{PSL}(2, q)$ is generated by elements of order $p$ and again we complete the proof in this case.

\section{References}

[1] A. Borel, Compact Clifford-Klein forms of symmetric spaces, Topology 2 (1963), 111-122.

[2] A. Borel and Harish-Chandra, Arithmetic subgroups of algebraic groups, Annals of Math. 75 (1962), 485-535.

[3] D. Coulson, O. Goodman, C. Hodgson and W. Neumann, Computing arithmetic invariants of 3-manifolds, Experimental Math. 9 (2000), 127 - 152.

[4] M. W. Davis, A hyperbolic 4-manifold, Proc. Amer. Math. Soc. 93 (1985), 325-328.

[5] M. Gromov and I. Piatetski-Shapiro, Nonarithmetic groups in Lobachevsky spaces, Publ. I.H.E.S. 66 (1988), 93-103.

[6] M. Gromov, H. Lawson and W. Thurston, Hyperbolic 4-manifolds and conformally flat 3-manifolds, Publ. I.H.E.S 68 (1988), 27-45.

[7] N. Kuiper, Hyperbolic 4-manifolds and tessellations, Publ. I.H.E.S 68 (1988), 47-76 (1989).

[8] G. W. Gibbons, Tunneling with a negative cosmological constant, Nuclear Phys. B 472 (1996), 683-708.

[9] S. Lang, Algebraic Number Theory, G.T.M. 110, Springer-Verlag, (1986).

[10] D. D. Long, Immersions and embeddings of totally geodesic surfaces, Bull. London Math. Soc. 19 (1987), 481-484.

[11] D. D. Long and A. W. Reid, On the geometric boundaries of hyperbolic 4-manifolds, Geometry and Topology, 4 (2000), 171-178.

[12] M. V. Nori, On subgroups of $\mathrm{GL}_{n}\left(\mathbf{F}_{p}\right)$, Invent. Math. 88 (1987), 257-276.

[13] W. Narkiewicz, Algebraic Numbers, Polish Scientific Publishers, Warsaw, 1974.

[14] O. T. O'Meara, Introduction to Quadratic Forms, Grundlehren der mathematischen Wissen. 117, Springer-Verlag, (1971).

[15] V. Platonov and A. Rapinchuk, Algebraic Groups and Number Theory, Academic Press (1994).

[16] M. S. Raghunathan, Discrete Subgroups of Lie Groups, Ergebnisse der Mathematik und ihrer Grenzgebiete, 68 Springer-Verlag (1972). 
[17] J. G. Ratcliffe, Foundations of Hyperbolic Manifolds, G.T.M. 149, Springer-Verlag, (1994).

[18] J. G. Ratcliffe and S. T. Tschantz, Gravitational instantons of constant curvature, Classical and Quantum Gravity 15 (1998), 2613-2627.

[19] M. Suzuki, Group Theory I, Grundlehren der mathematischen Wissen. 247, SpringerVerlag, (1982).

[20] W. P. Thurston, Three-dimensional manifolds, Kleinian groups and hyperbolic geometry, Bull. A.M.S. vol. 6 (1982), 357 - 381.

[21] E. B. Vinberg, Rings of definition of dense subgroups of semisimple linear groups, Math. USSR Izvest. 5 (1972), 45-55.

[22] J. Weeks, Topological questions in cosmology, to appear in the Proceedings of the Oklahoma conference on computational topology.

[23] B. Weisfeiler, Strong approximation for Zariski dense subgroups of semi-simple algebraic groups, Annals of Math. 120 (1984), 271 - 315.

[24] See http://www.math.ucsb.edu/ long/eta_file

Department of Mathematics, University of California Santa Barbara, CA 93106.

E-mail address: long@mail.math.ucsb.edu

Department of Mathematics, University of Texas Austin, TX 78712.

E-mail address: areid@math.utexas.edu 